\title{
Generating Compact Geometric Track-Maps for Train Positioning Applications
}

\author{
Hanno Winter*, Stefan Luthardt", Volker Willert*, Jürgen Adamy*
}

\begin{abstract}
In this paper, we present a method to generate compact geometric track-maps for train-borne localization applications. Therefore, we first give a brief overview on the purpose of track maps in train-positioning applications. It becomes apparent that there are hardly any adequate methods to generate suitable geometric track-maps. This is why we present a novel map generation procedure. It uses an optimization formulation to find the continuous sequence of track geometries that fits the available measurement data best. The optimization is initialized with the results from a localization filter [1] developed in our previous work. The localization filter also provides the required information for shape identification and measurement association. The presented approach will be evaluated on simulated data as well as on real measurements.
\end{abstract}

\section{INTRODUCTION}

For safety reasons, trains currently operate strictly signalbased. Therefore, each track is divided in multiple block sections delimited by signals. The signals are coordinated by a central safety logic which guarantees that only one train can occupy a block section at the same time. The necessary train-position information is gathered by sensors installed at the tracks. Although this system has proven to be safe and reliable it suffers either from high costs for the huge amount of sensors, or a low track capacity due to longer block sections [2]. To overcome this undesirable trade-off in the near future, trains have to become intelligent vehicles which are able to localize themselves continuously without any track-side installations.

The challenge when developing such a train-borne localization system is to fulfill the high demands in terms of reliability, availability, maintainability, and safety (RAMS) in the sense of EN 50126 [3]. Although the development of train-borne localization systems has gained of interest in recent years, there is currently no sensor configuration available fulfilling all demands [4].

In this paper, we want to focus on the purpose of digital track-maps in train-borne localization systems and how they can help to fulfill the RAMS demands in the near future. Therefore, we will investigate how track maps are utilized to improve the positioning accuracy, availability and integrity of train-borne localization systems. After that, we will give a brief overview over the methods commonly used to generate track maps. From this overview it will become apparent that there are hardly any adequate methods to generate suitable track maps for the purposes described above. Therefore, we will present a new approach to generate compact geometric

\footnotetext{
*Control Methods and Robotics Laboratory, TU Darmstadt, Germany \{hanno.winter,stefan.luthardt,vwillert,adamy\}@rmr.tu-darmstadt.de
}

track-maps based on the results of a localization filter we presented in our previous work [1].

\section{Track Maps in Train-Borne Localization}

We start with a brief overview on the different types of track maps and the methods used to generate them. Afterwards, we briefly discuss the shortcomings of these methods which motivated us to come up with a novel approach to generate compact geometric track-maps.

\section{A. Map Types}

There are three different categories of track maps used for train-borne localization:

1) Topological Track-Maps: This is the most basic trackmap type. It only stores the topology and mileage of the railway network. This is sufficient due to the fact that the position $p$ of a train can unambiguously be defined in railway coordinates by $p=\{t, s\}$, where $t$ represents a unique track ID and $s$ being a continuous track-length parameter. These maps are widely used in the railway system today since an additional absolute position information is not needed for its safe operation.

Theoretically, it is possible to realize a train-borne localization system with these maps. Therefore, it has to be assumed that the start point and the pre-set route of a specific train is known. Then a train can localize itself by measuring its traveled distance relative to its start point and thereby determining its position on this pre-set route [5]. Unfortunately, the pre-set route is normally not known on the train itself. This makes the localization result ambiguous: After a switch the position can no longer be clearly determined. To solve this ambiguity, maps holding additional information have been introduced as described next.

2) Topographic and Geometric Track-Maps: Compared to the topological track-maps described before, topographic track-maps additionally store the track-course in absolute coordinates. Furthermore, if they hold track-characteristic information like the specific track element type (straight, circular arc or transitional arc), orientation, curvature, or something similar, they can be additionally named geometric track-maps. The additional information stored in these maps allows to apply different map-matching techniques with more track-selective localization approaches compared to topological track-maps.

The map-matching approaches vary depending on the used sensor configuration. Many approaches utilize global navigation satellite system (GNSS) data and inertial measurement unit (IMU) data together with course and curvature 
information from a track map to realize track-selective mapmatching [6]-[10]. However, the additional map information can not only be used to improve the localization accuracy. It can also be used to increase the availability and integrity of the localization system itself [11]-[13].

3) Feature Track-Maps: This type of track-map also stores information on features or landmarks along the track. Features directly used for train-borne localization are for example ferromagnetic inhomogeneities of the rails [14] or characteristic distortions of the earth magnetic-field along the railway track [15]. Other features may be characteristic infrastructure elements like bridges, tunnels or stations, as suggested in [16] which can help to increase the accuracy and availability of GNSS positioning results.

\section{B. Generation Methods}

There are basically four main approaches to create digital track-maps [16]:

- Extraction from existing site plans, available as paper drawings, Computer Aided Design (CAD) plans, or Geographic Information System (GIS) databases,

- direct surveying of the tracks, e.g. by GNSS measurements or the application of tachymetry,

- analysis of orthophotos, or

- the application of simultaneous localization and mapping (SLAM) methods.

Although track maps are indispensable for train-borne localization, it is often not described in detail how the necessary maps are created. The probably most commonly used maps consist of previously recorded position data-points which are available from the localization sensors. If additional geometric track information is needed, it is mostly referred to the possibility to extract this data from existing site plans. To our knowledge there are currently two simultaneous localization and mapping (SLAM) approaches available which are especially designed for railway vehicles [17], [18]. Both methods create data-point based track maps. In the following, we present a new method that is not based on data points but on a concatenation of geometric entities.

\section{Conclusions for Track-Maps and their Generation}

Based on the explanations in Sec. II-A it becomes obvious how important track-maps are for train-borne localization. They help to increase the positioning accuracy, availability and integrity of the localization system. Thus, track maps act like an additional passive sensor helping to meet the RAMS requirements. This especially applies to geometric and feature track-maps. However, it should also be stressed that an inaccurate track map can also pose a single point of failure in the overall localization process.

The usability of map information for localization purposes is largely influenced by the map representation and the map quality. For a map to be suitable for train-borne localization it has to fulfill at least two basic requirements ${ }^{1}$ :

\footnotetext{
${ }^{1}$ Some further conclusions on the requirements for digital track-maps as well as some modeling schemes can be found in [16], [19], [20].
}

- Track-length accuracy: It is essential to consistently assign all stored information with respect to the tracklength $s$ since all localization algorithms somehow rely on this assignment.

- Compactness: All information must be accessible in a computationally efficient way, as the map is often directly used in the localization algorithm itself, which has to run in real-time. Furthermore, it is advantageous if the map consumes as little memory as possible in order to be easily transferable.

All current generation methods directly utilizing measurement data store the map in a data-point format. Between neighboring data points interpolation techniques are applied. To avoid large interpolation errors the tracks are normally densely sampled, i. e. with a sample distance between $1 \mathrm{~m}$ and $30 \mathrm{~m}$. Due to the necessary interpolation, such maps are not computationally efficient and the resulting map representation is neither easy accessible nor memory saving. Thus, these maps are not optimal in the sense of the compactness requirement mentioned above [21]. A more suitable track map representation would be a direct description of the geometric properties of each track element in a list. This would result in geometric track-maps easily fulfilling the compactness requirement. Those maps may be extracted from existing site plans. However, these site plans can differ significantly from the real track situation [22]. Two possible ways to create compact geometric track-maps based on measurement data are presented in [22], [23]. In the remainder of this paper an alternative mapping approach is presented, which generates a compact geometric track-map with a much simpler method. It advantageously incorporates the results of our previously published localization filter [1], and is furthermore more suitable for train-borne localization applications.

\section{MAP GENERATION}

The aim of our map generation procedure is to create compact geometric track-maps like the example listed in Tab. I. This table fully represents the track shown in Fig. 1. The compactness results from the fact that railway tracks always consist of a continuous sequence of well described geometric shapes (straight, transitional arc, and circular arc) [24]. Therefore, a railway track can unambiguously be described by a single starting point, the direction of the track at the starting point, the sequence of geometric shapes, and the geometric parameters for each shape (c.f. Tab. I).

TABLE I

COMPACT GEOMETRIC TRACK-MAP FOR THE TRACK SHOWN IN FIG. 1.

\begin{tabular}{|r||c|c|c|c|c|c|c|c|c|}
\hline Track ID & 1 & 2 & 3 & 4 & 5 & 6 & 7 & 8 & 9 \\
\hline Shape $^{\mathrm{a}}$ & st & ta & ca & ta & st & ta & ca & ta & st \\
\hline Length $L$ in $\mathrm{m}^{1000}$ & 231 & 476 & 231 & 1000 & 108 & 206 & 108 & 1000 \\
\hline Radius $^{\mathrm{b}} r$ in $\mathrm{m}$ & $\infty$ & -900 & -900 & -900 & $\infty$ & 300 & 300 & 300 & $\infty$ \\
\hline Start point & \multicolumn{5}{c|}{$\boldsymbol{p}_{\mathbf{0}}=\left[\begin{array}{ll}0 & 0\end{array}\right]^{\mathrm{T}} \varphi_{0}=10^{\circ}$} \\
\hline
\end{tabular}

a st: straight, ta: transitional arc, ca: circular arc

${ }^{\mathrm{b}}$ the sign indicates the turning direction ( + right, - left) 


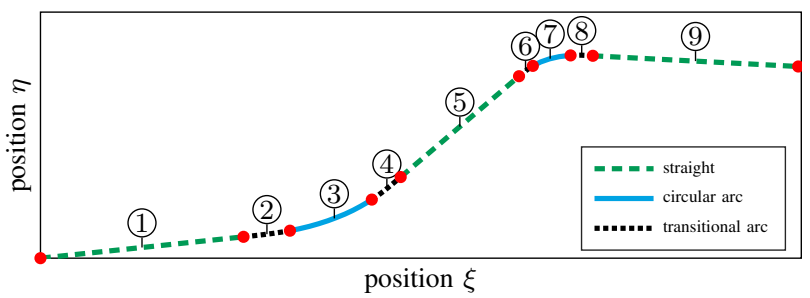

Fig. 1. Exemplary track consisting of the three standard track geometries: straight, transitional arc and circular arc. A compact geometric track-map representation of this track is given in Tab. I.

\section{A. Initial Situation}

We assume to start with the results of the localization filter we presented in [1]. Along with the position solution this filter estimates some of the track's geometric parameters which conveniently serve as initialization for the map generation process. Moreover, the filter delivers assignments between measurement data and identified track geometries which vastly simplifies the formulation of the mapping error that is derived later in this section.

A summary of the available parameters from the localization filter is listed in Tab. II. A visualization of the resulting discontinuous track is shown in Fig. 2. For this example, the input measurements used for the filter have been generated by simulation. The used ground-truth track is shown in Fig. 1. The detailed simulation procedure and parameters are described in [1]. All further explanations are illustrated using this example data.

\section{TABLE II}

INITIALLY AVAILABLE GEOMETRIC TRACK-MAP PARAMETERS

\begin{tabular}{|c|c|c|c|c|c|c|c|c|c|}
\hline Track ID & 1 & 2 & 3 & 4 & 5 & 6 & 7 & 8 & 9 \\
\hline Shape $^{\mathrm{a}}$ & st & - & $\mathrm{ca}$ & 一 & st & 一 & $\mathrm{ca}$ & - & st \\
\hline Length $L$ in $\mathrm{m}$ & 1035 & 278 & 415 & 206 & 983 & 185 & 106 & 165 & 962 \\
\hline Radius $^{\mathrm{b}} r$ in $\mathrm{m}$ & $\infty$ & - & -882 & - & $\infty$ & - & 297 & - & $\infty$ \\
\hline Start point $\xi_{0}$ in $\mathrm{m}$ & 0 & 1019 & 1306 & 1635 & 1772 & 2338 & 2480 & 2580 & 2763 \\
\hline Start point $\eta_{0}$ in $\mathrm{m}$ & 0 & 180 & 259 & 504 & 683 & 1487 & 1631 & 1663 & 1660 \\
\hline Direction $\varphi_{0}$ in ${ }^{\circ}$ & 10.0 & 10.0 & 23.2 & 50.1 & 54.9 & 54.9 & 27.9 & 7.4 & -4.8 \\
\hline
\end{tabular}

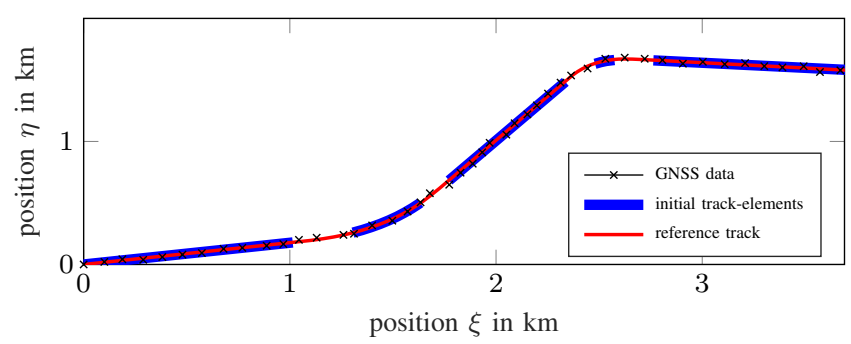

Fig. 2. Initial track-elements identified by the localization filter presented in [1]. These elements do not constitute a continuous track-map.

\section{B. Mapping Procedure}

The initial track depicted in Fig. 2 is not usable for localization since it is discontinuous. It has some gaps at the points where no track-geometry has been identified (c.f.
Tab. II, track-IDs: 2, 4, 6, and 8). Therefore, the task of the map generation procedure is to connect the initially identified track-elements to a continuous track which also has to fit the available GNSS measurement data. To solve this task, first, missing track-geometries have to be identified. Afterwards, the geometric parameters of the individual track-elements can be estimated.

1) Track Geometry Identification: We assume that the missing track-geometries can be concluded from the following knowledge [1], [24]: Railway tracks only consist of three basic geometric shapes which are straights, transitional arcs and circular arcs. A straight can only be connected to a circular-arc with the help of a transitional-arc and vice versa. Since the localization filter already identified straights and circular-arcs it can be concluded that the unknown geometries have to be transitional arcs.

2) Geometry Parameter Identification: After all track geometries have been identified, the corresponding geometric parameters have to be tuned such that the continuous concatenation of all track-elements fits best to the available GNSS measurement data. Although there is already a lot of information available from the localization filter, it is necessary to revise the parameters altogether. This can be seen when we try to simply concatenate all identified tracks. The parameters of the transitional arcs are inferred from the neighboring elements ${ }^{2}$. The resulting track-map for this

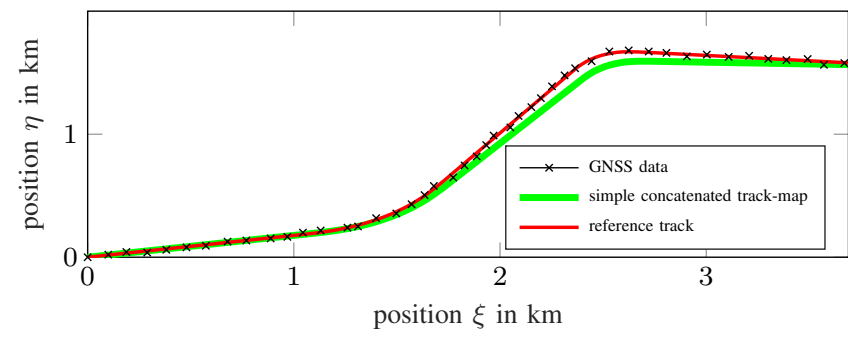

Fig. 3. Track-map resulting from the simple concatenation of the trackelements identified by the localization filter described in [1] (c.f. Tab. II and Fig. 2).

simple approach is shown in Fig. 3. Obviously, it is a quite poor fit to the GNSS data. This is a result of the continuity condition of railway tracks. Slight parameter inaccuracies of one track element are propagated on all succeeding elements. Therefore, it is necessary to tune the parameters in a joint optimization. To achieve this, we establish an optimization problem for the whole track by defining an appropriate error function that incorporates all given measurement information. In order to solve this optimization problem we furthermore have to reformulate the track parameters in a more suitable representation and have to choose an optimization method. The essential aspects of the optimization are described in the following paragraphs.

a) Error-Function Definition: The error introduced by a track element is given by the perpendicular distances between the track and the GNSS measurements related to this track

\footnotetext{
${ }^{2}$ This is possible because transitional arcs are built as clothoids [24]. They are clearly defined by their length, their radius in the end point, and their orientation either in the start or end-point.
} 
element. Let $\boldsymbol{x}_{t}$ be a vector representing the parameters of track element $t$. Furthermore, let $\boldsymbol{z}_{t, i}$ be the $i$-th GNSS position measurement assigned to this track element $t$ by the localization filter. With $\hat{\boldsymbol{z}}_{t, i}\left(\boldsymbol{x}_{t}\right)$, the dropped perpendicular point of $z_{t, i}$ on the track element $t$, the error for this measurement is then defined as

$$
\boldsymbol{e}_{t, i}\left(\boldsymbol{x}_{t}\right)=\boldsymbol{z}_{t, i}-\hat{\boldsymbol{z}}_{t, i}\left(\boldsymbol{x}_{t}\right)
$$

The sum over all this measurement errors for all trackelements $\mathcal{T}$ yields the total error of the whole track map, i.e.

$$
F\left(\boldsymbol{\mathcal { X }}=\left\{\boldsymbol{x}_{1}, \ldots, \boldsymbol{x}_{N}\right\}\right)=\sum_{t \in \mathcal{T}} \sum_{i \in \mathcal{C}_{t}} \boldsymbol{e}_{t, i}^{\mathrm{T}} \boldsymbol{\Omega}_{t, i} \boldsymbol{e}_{t, i},
$$

where $N$ is the number of identified track-geometries, $\mathcal{C}_{t}$ is the set of all measurements assigned to track $t$ and $\boldsymbol{\Omega}_{t, i}$ is the information matrix corresponding to measurement $\boldsymbol{z}_{t, i}$. The information matrix is the inverse of the covariance matrix which is often provided by the GNSS receiver. If no adequate uncertainty information is available $\Omega_{t, i}$ should be chosen according to the assumed receiver uncertainty.

b) Parameter Representation: The parameterization of the track map presented in Tab. I is not very suitable for an optimization. With this parameterization the whole track would be very sensitive to changes in specific parameters, e. g. small changes in $\varphi_{0}$ or $L$, would rotate, respectively move, major parts of the track. Therefore, an alternative representation is chosen with less sensitivity. All straights are now parameterized by their start and end point whereas transitional arcs and circular arcs are parameterized by a minimal set of geometric parameters. For our example track (c.f. Tab. II) this new parameterization is given in Tab. III and the corresponding parameter vector is

$$
\begin{aligned}
\mathcal{X}= & \left\{\boldsymbol{x}_{1}, \boldsymbol{x}_{2}, \boldsymbol{x}_{3}, \boldsymbol{x}_{4}, \boldsymbol{x}_{5}, \ldots\right\}, \text { with } \\
\boldsymbol{x}_{1} & =\left[\begin{array}{llll}
\xi_{0,1} & \eta_{0,1} & \xi_{e, 1} & \eta_{e, 1}
\end{array}\right]^{\mathrm{T}}, \\
\boldsymbol{x}_{2} & =L_{2}, \quad \boldsymbol{x}_{3}=\left[\begin{array}{lll}
r_{3} & L_{3}
\end{array}\right]^{\mathrm{T}}, \quad \boldsymbol{x}_{4}=L_{4}, \\
\boldsymbol{x}_{5} & =\left[\begin{array}{llll}
\xi_{0,5} & \eta_{0,5} & \xi_{e, 5} & \eta_{e, 5}
\end{array}\right]^{\mathrm{T}}, \quad \ldots .
\end{aligned}
$$

TABLE III

TRACK-MAP FROM TAB. II REPARAMETERIZED FOR THE OPTIMIZATION.

\begin{tabular}{|r|c|c|c|c|c|c|c|c|c|}
\hline Track ID & 1 & 2 & 3 & 4 & 5 & 6 & 7 & 8 & 9 \\
\hline Shape $^{\mathrm{a}}$ & st & ta & ca & ta & st & ta & ca & ta & st \\
\hline Length $L$ in $\mathrm{m}^{\prime}$ & - & 278 & 415 & 206 & - & 185 & 106 & 165 & - \\
\hline Radius $^{\mathrm{b}} r$ in $\mathrm{m}$ & - & - & -882 & - & - & - & 297 & - & - \\
\hline Start point $\xi_{0}$ in $\mathrm{m}$ & 0 & - & - & - & 1772 & - & - & - & 2763 \\
\hline Start point $\eta_{0}$ in $\mathrm{m}$ & 0 & - & - & - & 683 & - & - & - & 1660 \\
\hline End point $\xi_{e}$ in $\mathrm{m}$ & 1019 & - & - & - & 2338 & - & - & - & 3722 \\
\hline End point $\eta_{e}$ in $\mathrm{m}$ & 180 & - & - & - & 1487 & - & - & - & 1579 \\
\hline
\end{tabular}

a st: straight, ta: transitional arc, ca: circular arc

$\mathrm{b}$ the sign indicates the turning direction (+ right, - left)

c) Optimization Method: The objective is to minimize the error function $F(\mathcal{X})$ given in (2). Although the initial parameters given by the localization filter yield a poor track map when being simply concatenated (c.f. Fig. 3), they are still a good initial guess $\mathcal{X}_{0}$ for the track-map parameters. Therefore, we can start the optimization with this initial parameter set which is presumably close to the global optimum and it is sufficient to use the Levenberg-Marquardt algorithm [25] to find that optimum.

\section{EVAluATION}

In this section the performance of the presented mapping method will be evaluated with the help of simulated data and real measurement data.

\section{A. Simulation Results}

First, an evaluation based on simulations is carried out to gain some principal insights into the behavior of the presented mapping algorithm. This is advantageous, as in simulations ground-truth data is directly available. Throughout all simulations the example track described in Sec. III-A is used.

1) Optimization Process: The progress of the residual $\|F(\mathcal{X})\|$ during the optimization is shown in Fig. 4. It can be seen that the optimization converges very fast. After eight iterations the stopping criteria (relative step size limit of $1 \mathrm{e}-6$ ) is reached. The biggest change in $\|F(\mathcal{X})\|$ and the parameter set occurs in the first iteration. This confirms our hypothesis that the initial parameter set $\mathcal{X}_{0}$ provided by the localization filter, is already a good guess and the Levenberg-Marquardt algorithm can quickly find a good solution.

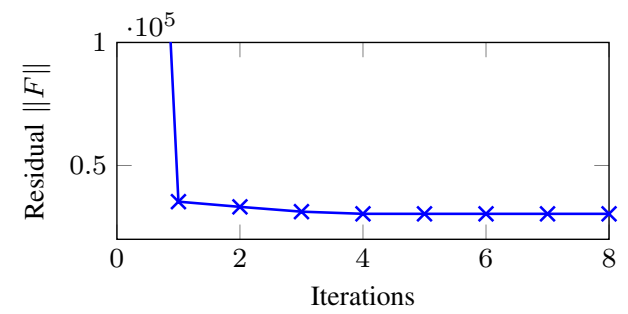

Fig. 4. Simulation result: Progress of the residual during the optimization.

2) Absolute Accuracy: A visualization of the resulting track is shown in Fig. 5. A good qualitative match with the GNSS measurements and the reference track becomes evident. Figure 6 allows to investigate the map's quality in even more detail. The plot shows the absolute position deviation to the reference track over the path length $s$. The generated map is compared to a typically used data-point based map which has been sampled from the virtually generated GNSS data with a spacing of $1 \mathrm{~m}$. Intermediate points are calculated by a linear interpolation. The deviation of our optimized geometric track-map to the reference track is on average $1.8 \mathrm{~m}$. The error of the data-point based map varies strongly over the whole track length and the average deviation is $10.3 \mathrm{~m}$. This value corresponds with the simulated GNSS measurement noise which has a standard deviation of $10 \mathrm{~m}$.

The better performance of the new approach results from the joint incorporation of all measurements in the optimization process. Thereby, the error induced by the GNSS measurement noise can be reduced significantly. 


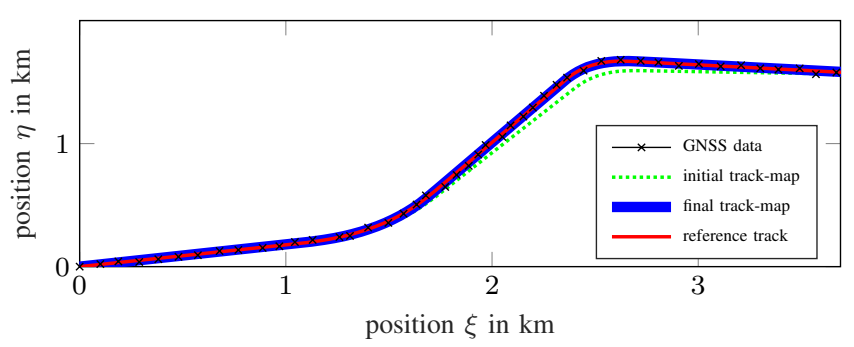

Fig. 5. Simulation result: Final geometric track-map resulting from the here presented mapping approach. Due to the optimization procedure the final map fits very well to the GNSS data, compared to the initial map resulting from the simple concatenation of the identified track elements.

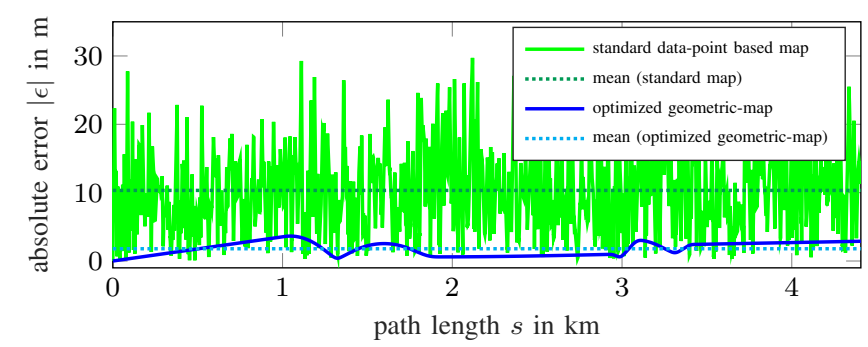

Fig. 6. Simulation result: Absolute mapping error $|\epsilon|$ plotted against the track length $s$. The error of the geometric track-map generated with the here presented approach is significantly smaller compared to a typically used data-point based track map.

3) Geometric Accuracy: An often used metric to evaluate the geometric similarity of two paths is the fréchet distance [26]. The fréchet distance of the optimized geometric trackmap to the reference track is $3.6 \mathrm{~m}$. In comparison, the fréchet distance of the data-point based map is $26.9 \mathrm{~m}$. Consequently, the generated geometric track-map is significantly better in representing the geometric characteristic of the track. Furthermore, the optimized geometric track-map allows to efficiently access useful geometric information, e.g. the curvature at an arbitrary path length. For the data-point based map this is only possible with additional calculations.

4) Map Size: To compare the sizes of the maps, we express the storage demand as the number of necessary data fields. For example, a position specification $\boldsymbol{p}=(\xi \eta)^{T}$ requires two data fields. The data-point based map which is $4.4 \mathrm{~km}$ long and sampled at a density of $1 \mathrm{~m}$, therefore, consist of more than 8000 data fields, whereas the generated track-map only consists of 38 data fields. This clearly shows how compact the optimized geometric track-map is, compared to a simple data-point based map.

\section{B. Evaluation on Real Measurement Data}

The mapping performance is also evaluated with real GNSS and IMU measurement data. The data has been recorded on a $24 \mathrm{~km}$ long test drive on a secondary line in the Erzgebirge in Germany. The results presented next belong to a $5.7 \mathrm{~km}$ long section of this track.

1) Absolute Accuracy: The absolute accuracy of the optimized geometric track-map is evaluated with the help of OpenStreetMap (OSM) data [27] since no other reference is available. In Fig. 8 the cumulative distribution function (CDF) of the absolute mapping error $|\epsilon|$, i. e. the perpendicular distance between the track from the optimized geometric track-map and the OSM map, is shown.

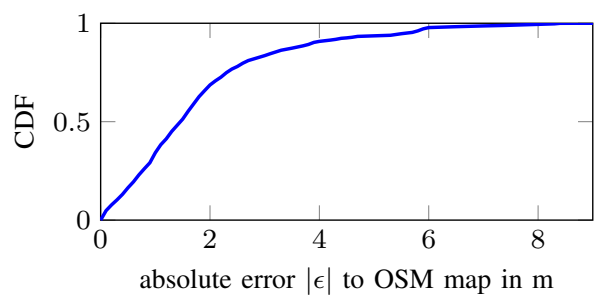

Fig. 7. Result on real measurement data: Cumulative distribution function (CDF) of the absolute mapping error $|\epsilon|$ between the optimized geometric track-map and the OSM map.

The mean error is $1.8 \mathrm{~m}$ and the maximum mapping error is $8.7 \mathrm{~m}$. For the optimized geometric track-map a mapping error of less than $2 \mathrm{~m}$ is achieved on $68 \%$ of the track (c. f. Fig. 7). It can be concluded that the generated map is quite accurate and that the presented mapping method is also applicable on real data. However, when projecting the OSM map on a satellite image, it can be seen that the OSM map sometimes gives a poor fit to the visible course of the rails. Interestingly, the biggest deviations between the optimized geometric trackmap and the OSM map occurs at these sections. Thus, it is very likely that the real error of the optimized geometric track-map is even smaller than stated above.

2) Geometric Accuracy: The geometric accuracy of the optimized geometric track-map is evaluated with the help of satellite images as shown in Fig. 8. As in the simulational

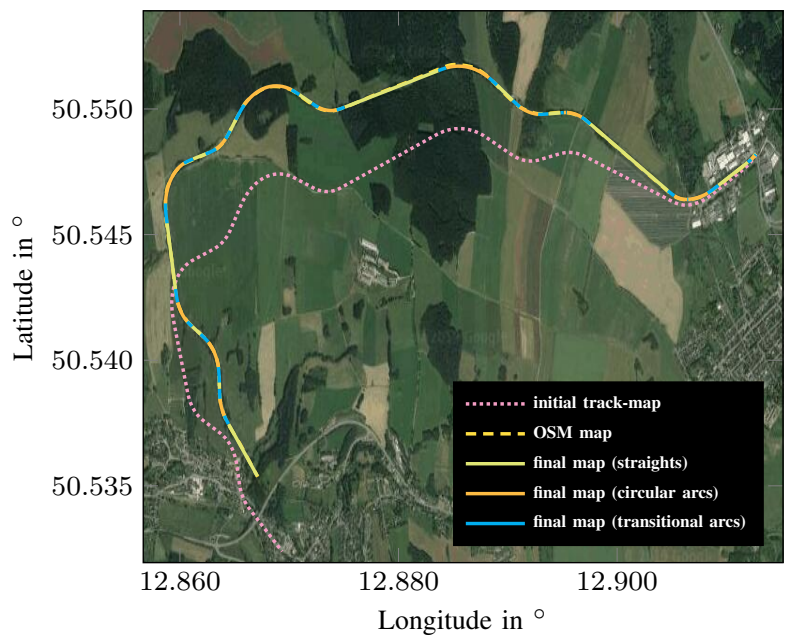

Fig. 8. Result on real measurement data: Visualization of the generated map on a satellite image ${ }^{4}$. The OSM map is not visible on this scale as it is perfectly covered from the generated map.

evaluation, it can be seen that the initial map, resulting from the simple concatenation of all identified track elements, yields a rather poor fit of the real track. Only the first track elements are near the course of the real track (see Fig. 8 considering the direction of travel from east to west). Due to the continuity condition, these small errors on the first track

\footnotetext{
${ }^{4}$ Image (c) 2019 Google, Maps (c) 2019 GeoBasis-DE/BKG (c) 2009), Google
} 
elements accumulate for the track elements further away of the start.

In contrast, the final map resulting from the optimization corresponds very well to the course of the rails visible on the satellite image (c.f. Fig. 8). Therefore, we assume that the geometric shape of the track has been mapped in a suitable way for train-borne localization applications.

\section{COnClusions}

In this paper we presented an approach to generate geometric track-maps for train-borne localization applications. After a brief overview of the existing track-map types and map generation methods, we argued how important track maps are for trains to become intelligent vehicles that are able to localize themselves in the near future. Furthermore, the overview revealed that there are hardly any adequate methods to generate suitable track maps for this purpose.

We presented an optimization method that finds the geometric parameters of a continuous track that fits the position measurements best and is much more compact. The method uses information provided by a localization filter for initialization, shape identification and data association.

Through a simulative evaluation we demonstrated that the presented method is able to generate geometric trackmaps which are more accurate than the typically used datapoint based maps. Furthermore, the generated map provides additional geometric track information and is much more compact. Finally, we demonstrated on a $5.7 \mathrm{~km}$ long real track-section that the approach is also applicable on real measurement data and the resulting map corresponds very well to the track visible in satellite images. Consequently, the presented method is capable of generating compact geometric track-maps, which can help to introduce trainborne localization systems in the near future.

\section{ACKNOWLEDGMENT}

We kindly thank Deutsche Bahn for supporting this research project. Furthermore, we like to thank Thales affording us to collect the raw data with their test vehicle LUCY, and the group of Geodetic Measurement Systems and Sensors at TU Darmstadt for providing the IMU/GNSS sensor platform.

\section{REFERENCES}

[1] H. Winter, V. Willert, and J. Adamy, "Increasing accuracy in train localization exploiting track-geometry constraints," in Proc. IEEE Int. Conf. on Intelligent Transportation Syst. (ITSC), Nov. 2018, pp. 15721579.

[2] G. Theeg and S. Vlasenko, Eds., Railway Signalling \& Interlocking: International Compendium, 1st ed. Eurailpress, 2009.

[3] EN50126 - Railway applications - The specification and demonstration of Reliability, Availability, Maintainability and Safety (RAMS), Eur. Commitee for Electrotechnical Standardization Std.

[4] J. Otegui, A. Bahillo, I. Lopetegi, and L. E. Díez, "A survey of train positioning solutions," IEEE Sensors J., vol. 17, no. 20, pp. 6788-6797, Aug. 2017.

[5] U. Schneider and J. Troelsen, "Introducing digital map information into train positioning systems: chances and risks," WIT Trans. on The Built Environment, vol. 50, 2000.

[6] S. S. Saab, "A map matching approach for train positioning. i. development and analysis," IEEE Trans. Veh. Technol., vol. 49, no. 2, pp. 467-475, Mar. 2000.
[7] K. Lüddecke and C. Rahmig, "Evaluating multiple GNSS data in a multi-hypothesis based map-matching algorithm for train positioning," in Proc. IEEE Intelligent Vehicles Symp. (IV), Jun. 2011, pp. 10371042 .

[8] A. Broquetas, A. Comerón, A. Gelonch, J. M. Fuertes, J. A. Castro, D. Felip, M. A. López, and J. A. Pulido, "Track detection in railway sidings based on MEMS gyroscope sensors," MDPI AG Sensors J., vol. 12, pp. 16228-16249, Nov. 2012.

[9] O. G. Crespillo, O. Heirich, and A. Lehner, "Bayesian GNSS/IMU tight integration for precise railway navigation on track map," in Proc. IEEE Position, Location and Navigation Symp. - PLANS. IEEE/ION, May 2014, pp. 999-1007.

[10] M. Roth, B. Baasch, P. Havrila, and J. Groos, "Map-supported positioning enables in-service condition monitoring of railway tracks," in Proc. IEEE Int. Conf. on Information Fusion (FUSION), Sep. 2018, pp. 2346-2353.

[11] A. Neri, V. Palma, F. Rispoli, and A. M. Vegni, "Track constrained PVT estimation based on the double-difference technique for railway applications," in Proc. IEEE Eur. Signal Process. Conf. (EUSIPCO), Sep. 2013, pp. 1-5.

[12] B. Yu and Y. Eun, "Sensor attack detection for railway vehicles using topographic information," in Proc. IEEE Int. Conf. on Control, Automation and Systems (ICCAS), Oct. 2017, pp. 149-154.

[13] C. Jin, B. Cai, J. Wang, and A. Kealy, "DTM-aided adaptive EPF navigation application in railways," MDPI AG Sensors J., vol. 18, no. 11 , Nov. 2018

[14] M. Spindler and M. Lauer, "High accuracy estimation of velocity and position for railway vehicles using ferromagnetic inhomogeneities," in Proc. IEEE Int. Conf. on Intelligent Transportation Syst. (ITSC), Nov. 2018, pp. 1202-1207.

[15] B. Siebler, O. Heirich, and S. Sand, "Train localization with particle filter and magnetic field measurements," in Proc. IEEE Int. Conf. on Information Fusion (FUSION), Jul. 2018, pp. 1-5.

[16] K. Gerlach and M. M. zu Hörste, "A precise digital map for galileobased train positioning systems," in Proc. IEEE Int. Conf. on Intelligent Transport Systems Telecommunications (ITST), Oct. 2009, pp. 343-347.

[17] O. Heirich, P. Robertson, and T. Strang, "RailSLAM - localization of rail vehicles and mapping of geometric railway tracks," in Proc. IEEE Int. Conf. on Robotics and Automation (ICRA), May 2013, pp. 5212-5219.

[18] C. Hasberg, S. Hensel, and C. Stiller, "Simultaneous localization and mapping for path-constrained motion," IEEE Trans. Intell. Transp. Syst., vol. 13, no. 2, pp. 541-552, Jun. 2012.

[19] G. Bikker, K.-A. Klinge, M. Schroeder, and E. Schnieder, "Concepts of an intelligent route atlas for localisation in guided traffic," in Proc. DGON Int. Conf. on Land Vehicle Navigation (EURNAV), Jun. 1998, pp. $157-165$.

[20] F. Böhringer and A. Geistler, "Location in railway traffic: Generation of a digital map for secure applications," WIT Trans. on The Built Environment, pp. 459-468, 2006.

[21] J. Liu, B. Cai, J. Wang, and Z. Yang, "Generating an electronic track map for train collision early warning system," in Proc. IEEE Int. Conf. on Intelligent Rail Transportation (ICIRT), 2013, pp. 140-145.

[22] W. Li, H. Pu, P. Schonfeld, Z. Song, H. Zhang, L. Wang, J. Wang, $\mathrm{X}$. Peng, and L. Peng, "A method for automatically recreating the horizontal alignment geometry of existing railways," Wiley Online Library, Computer-Aided Civil and Infrastructure Engineering, vol. 34, no. 1, pp. 71-94, 2019

[23] W.-J. Tao, B.-G. Cai, J. Wang, J. Liu, and W. Shang-Guan, "Digital track map generation for safety-critical railway applications," in Proc. ION Int. Tech. Meeting of the Satellite Division of the Inst. of Navigation (GNSS), vol. 3, 2017, pp. 1978-1987.

[24] J. Haldor and F. Lademann, Planung von Bahnanlagen: GrundlagenPlanung-Berechnung, 2nd ed. Carl Hanser Verlag GmbH \& Co. KG, 2017.

[25] J. J. Moré, "The levenberg-marquardt algorithm: Implementation and theory," in Numerical Analysis, G. A. Watson, Ed. Springer Berlin Heidelberg, 1978, pp. 105-116.

[26] M. Kubicka, A. Cela, H. Mounier, and S.-I. Niculescu, "Comparative study and application-oriented classification of vehicular map-matching methods," IEEE Trans. Intell. Transp. Syst., vol. 10, no. 2, pp. 150-166, Apr. 2018.

[27] OpenStreetMap contributors, "Extract retrieved from https://overpassapi.de," https://www.openstreetmap.org, 2019. 